\title{
Oligodendrocytes Promote Neuronal Survival and Axonal Length by Distinct Intracellular Mechanisms: A Novel Role for Oligodendrocyte-Derived Glial Cell Line-Derived Neurotrophic Factor
}

\author{
Alastair Wilkins, Henry Majed, Robert Layfield, Alastair Compston, and Siddharthan Chandran \\ Cambridge Center for Brain Repair, Forvie Site, Cambridge CB2 2PY, United Kingdom
}

\begin{abstract}
Interactions of CNS cells lead to the establishment of complex neural systems. Specifically, oligodendrocytes form myelin sheaths around axons that enable rapid electrical conduction of impulses. Recent evidence has emerged that oligodendrocytes may also release trophic factors promoting neuronal survival. We therefore studied the effects of factors released from cells of the oligodendrocyte lineage on neuronal survival and also on the morphology of neurons. Neurons derived from rat embryonic cortices were cultured and exposed to media conditioned by oligodendrocyte precursor cells (OPCs) or differentiated oligodendrocytes. In line with previous studies, exposure of OPC and oligodendrocyte-conditioned media (OCM) increased survival, a phosphatidylinositol 3'-kinase (PI3kinase)/Akt-dependent phenomenon. In addition, exposure of neurons to OCM but not OPC conditioned media resulted in increased axonal length per neuron, as detected by antibodies to phosphorylated neurofilaments. OCM exposure resulted in activation of the MAPkinase/extracellular signalregulated kinase pathway, inhibition of which significantly reduced oligodendrocyte-mediated enhancement of axonal length but, unlike PI3kinase inhibition, had no effect on neuronal survival. Furthermore, we identify glial cell line-derived neurotrophic factor (GDNF) production by differentiated oligodendrocytes and provide evidence that implicates GDNF in OCM-mediated axonal effects, independent of its effect on neuronal survival. Therefore, we have shown that factors released by OPCs and oligodendrocytes induce the activation of distinct intracellular pathways within neurons, which have different functional effects on the cell.
\end{abstract}

Key words: oligodendrocyte; neuron; axon; PI3kinase/Akt; MAP kinase; trophic

\section{Introduction}

During the development of the CNS, approximately half of all neurons undergo apoptotic death (Raff et al., 1994). The survival and subsequent maintenance of neuronal integrity requires continued suppression of the intrinsic apoptotic machinery by, for example, target-derived neurotrophic mechanisms (Henderson, 1996). The precise nature of signals that mediate neuronal survival is complex and many factors activating various intracellular pathways have been implicated. Two pathways of importance, both to neuronal survival and process formation, are the phosphatidylinositol 3'-kinase (PI3kinase)/Akt and MAPkinase/extracellular signal-regulated kinase (Erk)-signaling pathways (Atwal et al., 2000).

The influence of oligodendrocytes on axonal calibre and function is well described. Oligodendrocytes myelinate axons, increase axonal stability, and induce local accumulation and phosphorylation of neurofilaments within the axon (Colello et al., 1994; Sanchez et al., 1996, 2000; Brady et al., 1999). Neuronal function is also influenced by oligodendrocyte-derived soluble factors that induce sodium channel-clustering along axons, necessary for efficient saltatory conduction, and maintain this clus-

\footnotetext{
Received Sept. 9, 2002; revised March 17, 2003; accepted March 18, 2003.

These studies were supported by The Vera and Carl Johan Michaelson Legacy, Denmark; The Sackler Fund, University of Cambridge (A.W.); and the Medical Research Council, United Kingdom (S.C.).

Correspondence should be addressed to Alastair Wilkins, Cambridge Center for Brain Repair, Forvie Site, Robinson Way, Cambridge CB2 2PY, UK. E-mail: aw255@hermes.cam.ac.uk.

Copyright $\odot 2003$ Society for Neuroscience $\quad$ 0270-6474/03/234967-08\$15.00/0
}

tering even in the absence of direct axon-glial contact (Kaplan et al., 1997). Despite the intimate association of oligodendrocytes and axons within the CNS, the contribution of direct glialmediated survival is comparatively understudied and neuronal survival has primarily been viewed to be a determinant of targetderived peptide factors.

A concept that emerged from the study of mechanisms that underlie peripheral neuronal survival is the requirement of Schwann cell-derived signals for peripheral neuronal survival (Riethmacher et al., 1997). The ability to grow enriched populations of CNS neurons in defined conditions permits the examination of similar relationships within the CNS. Indeed, recent evidence has emerged that oligodendrocytes may also provide direct trophic support for neurons, which raises the possibility that the loss of oligodendrocyte-derived factors may lead to neuronal death under certain situations (Meyer-Franke et al., 1995; Griffiths et al., 1998). Furthermore, survival requirements may differ within compartments of the cell with mechanisms of axonal degeneration differing from those affecting the cell body (Finn et al., 2000). The reciprocal relationship between glia and neurons raises the possibility that oligodendrocytes may influence neuronal function by mechanisms that are distinct from those necessary for survival.

We have shown previously that oligodendrocyte-derived soluble factors, of which insulin-like growth factor (IGF)-1 is a significant component, can promote the survival of developing cortical neurons (Wilkins et al., 2001). In the present study, we have 
characterized the signaling mechanisms that mediate oligodendrocyte-derived survival and assessed whether glial-derived factors influence axonal morphology as determined by neurofilament phosphorylation. We show that oligodendrocyte precursor cells (OPCs) and differentiated oligodendrocytes secrete factors that increase the survival of cultured embryonic cortical neurons via the PI3kinase/Akt-signaling pathway. In contrast, only differentiated oligodendrocyte-derived medium was observed to increase levels of phosphorylated neurofilaments per neuron, a MAPkinase/Erkdependent phenomenon. We provide evidence that differentiated oligodendrocyte-derived glial cell line-derived neurotrophic factor (GDNF) is responsible for the modulation of phosphorylated neurofilament levels. Although the MAPkinase/Erk pathway is well known to influence neurite/axonal process formation, the secretion of GDNF from oligodendrocytes and subsequent effects on neurofilament phosphorylation are novel.

\section{Materials and Methods}

\section{Cell culture}

Preparation of oligodendrocyte precursor cells. Mixed glial cell cultures were prepared following the protocol of McCarthy and deVellis (1980), as described previously (Wilkins et al., 2001). Briefly, forebrains of newborn rat pups were removed and the meninges stripped before mechanical and enzymatic dissociation. The resulting cell suspension was plated onto poly-L-lysine coated $75 \mathrm{~cm}^{2}$ tissue culture flasks. Culture medium (DMEM plus $10 \%$ fetal calf serum) was changed at $24 \mathrm{hr}$ and twice weekly thereafter until the cells reached confluence after 10-12 d. At this stage, a loosely adherent superficial layer of cells that represents OPCs and microglia lying on a confluent astrocyte layer was isolated by two-stage differential adhesion. Microglia were removed by vigorous shaking of the cultures at $240 \mathrm{rpm}$, followed by a change to fresh medium. Top-dwelling OPCs were dislodged by overnight shaking of the cultures at $160 \mathrm{rpm}$ and the supernatant was placed into uncoated tissue culture flasks for $30 \mathrm{~min}$ to allow adherence of any residual microglia. The loosely adherent OPCs were dislodged by gentle manual shaking. The final supernatant from these shaken cultures contained $\sim 85-90 \%$ OPCs. Cells were expanded in B104 conditioned medium to further enrich and expand the oligodendrocyte cultures (Cannoll et al., 1996).

Preparation of oligodendrocyte-conditioned medium. Enriched OPCs were plated at high density $\left(5 \times 10^{5}\right.$ per poly-L-lysine-coated six well plates) in serum-free DMEM supplemented with 2\% B27 (Invitrogen, Paisley, UK). At appropriate stages, cells were washed in DMEM and cultured in conditioning medium (DMEM plus insulin-free Sato) containing bovine serum albumin $(100 \mu \mathrm{g} / \mathrm{ml})$, transferrin $(100 \mu \mathrm{g} / \mathrm{ml})$, progesterone $(0.06 \mu \mathrm{g} / \mathrm{ml})$, putrescine $(16 \mu \mathrm{g} / \mathrm{ml})$, selenite $(0.04 \mu \mathrm{g} / \mathrm{ml})$, thyroxine $(0.04 \mu \mathrm{g} / \mathrm{ml})$, and triiodothryonine $(0.04 \mu \mathrm{g} / \mathrm{ml})$ for $24 \mathrm{hr}$. After centrifugation at $1000 \mathrm{rpm}$ for $5 \mathrm{~min}$ and passage through a $45 \mu \mathrm{m}$ filter, the resulting supernatant was stored at $-20^{\circ} \mathrm{C}$ and diluted $1: 1$ with fresh medium for experiments. Astrocyte conditioned medium (ACM) was prepared using purified primary astrocyte derived from mixed glial cultures (plated at $5 \times 10^{5}$ per poly-L-lysine coated six well plates in DMEM plus $10 \%$ fetal calf serum and conditioned as above).

Neuronal cell culture. Neuronal cell cultures were prepared from cortices of embryonic day (E) 16 rat embryos as described previously (Wilkins et al., 2001). After enzymatic dissociation, cells were plated onto poly- L-lysine-coated $13 \mathrm{~mm}$ coverslips and cultured in DMEM supplemented with 2\% B27 or Sato supplemented DMEM (as above). Recombinant IGF-1 was obtained from Roche (Lewes, UK). The pathway inhibitors LY294002 and wortmannin were obtained from Sigma (Poole, UK) and PD98059 was obtained from Calbiochem (La Jolla, CA). AntiIGF-1-blocking antibodies were obtained from Upstate Biotechnology (Lake Placid, NY) and anti-GDNF-blocking antibodies were obtained from R \& D Systems (Minneapolis, MN).

\section{Immunocytochemistry}

Immunocytochemistry was used to identify cell phenotypes. Cells were stained "live" or after fixation with $4 \%$ paraformaldehyde. The following primary antibodies against cell surface markers were used: 1:4 galactoce- rebroside (GalC); 1:4 A2B5, and 1:10 O4 (all derived from hybridoma lines; European Collection of Cell Cultures, Salisbury, UK). The following primary antibodies against intracellular markers were used after treatment of fixed cultures with $100 \%$ methanol for 10 min at $-20^{\circ} \mathrm{C}$ : 1:400 $\beta$-tubulin III (Sigma); 1:50 glial fibrillary acidic protein (GFAP) (Dako, Ely, UK); 1:10 terminal deoxynucleotidyl transferase-mediated biotinylated UTP nick end labeling (TUNEL) (Boehringer); 1:1000 lectin from 1:100 Bandiera simplicifolia BS-1 (Sigma); 1:100 SMI312 (phosphorylated neurofilaments) (Sternberger Monoclonals, Exeter, UK); and anti-GDNF (Santa Cruz Biotechnology, Santa Cruz, CA). Secondary antibodies coupled to fluorochromes FITC, tetramethylrhodamine isothiocyanate, or 7-amino-4-methylcoumarin-3 acetic acid were used to visualize primary antibody staining. Hoechst 33258 (1:5000 bisbenzamide) was used (10 min at room temperature) for nuclear identification.

\section{Assays for cell survival and cell morphology}

Evaluation of cell survival and morphology within cultures was performed by the following methods.

Morphological analysis using immunocytochemistry. Neuronal cells were identified by $\beta$-tubulin expression. In addition, nuclear staining (Hoechst) of cells enabled a morphological assessment of apoptosis. Neuronal survival was assessed using counts of live $\beta$-tubulin positive stained cells as the "gold-standard." In all cases, a control culture of cells grown throughout the experimental period in DMEM/2\% B27 was also analyzed and values for experimental conditions were divided by this value to standardize results between experiments. TUNEL staining was subsequently undertaken to confirm apoptotic cell death.

Analysis of axons in culture. The antibody SMI312 (Sternberger) labels phosphorylated neurofilaments and can be used to distinguish axons from dendrites (Lee et al., 1987). Cultures were stained for SMI312 after fixation and were viewed under a fluorescent microscope with digital images $(40 \times)$ taken of three random fields within each culture (at least three culture conditions per experiment). The images were analyzed using Scion (Frederick, MD) imaging software. Lengths of processes staining for SMI312 per field were obtained, together with the number of live cells per field. The ratio of SMI312 length to cell number therefore gave an index of phosphorylated neurofilament length per live cell.

\section{Immunoblotting for cell signaling proteins and growth factors}

Neurons were cultured at high density $\left(2 \times 10^{6}\right.$ per six well plate $)$ before exposure to test condition. After $30 \mathrm{~min}$, cells were lysed in $150 \mathrm{~mm}$ Tris-HCl, $8 \mathrm{M}$ urea, 2.5\% w/v SDS, $20 \% \mathrm{v} / \mathrm{v}$ glycerol, $10 \% \mathrm{v} / \mathrm{v}$ 2-mercaptoethanol, 3\% w/v DTT, $0.1 \%$ bromophenol blue, $\mathrm{pH}$ 6.8, and stored at $-20^{\circ} \mathrm{C}$ until use. Continuous gradient SDS-polyacrylamide gels (5-20\%) were used and equal volumes of cell lysates [equivalent to equal amounts of total protein as judged by protein determination using the BCA protein assay kit (Pierce, Cheshire, UK)] were run on each lane. After transfer to nitrocellulose membrane (Hybond C-super; Amersham Biosciences, Amersham, UK) and blocking in $4 \% \mathrm{w} / \mathrm{v}$ powdered milk, membranes were incubated overnight in primary antibody at $4^{\circ} \mathrm{C}$. Antibodies used were phospho-Akt (Ser473-specific), Akt antibody, phospho-MAPkinase p42/p44, and nonphosphorylated MAPkinase p42/ p44 (all 1:1000; New England Biolabs, Beverly, MA). Immunoreactivity was visualized by secondary anti-rabbit HRP-conjugated antibodies (New England Biolabs) and enhanced chemoluminescence (Renaissance ECL reagent; NEN, Boston, MA). When necessary, membranes were stripped (Restore Western Blot stripping buffer; Pierce) and reblotted.

Immunoblotting for growth factors was performed in a similar way. Conditioned media were concentrated $(100 \times)$ using $3 \mathrm{kDa}$ cutoff filters (Millipore, Bedford, MA) before loading on $12.5 \%$ SDS-polyacrylamide gels. Gels were transferred and blotted for antibodies to insulin such as growth factor type 2 (IGF-2; Upstate Biotechnology), nerve growth factor (NGF- $\beta$ ), ciliary neurotrophic factor (CNTF), GDNF, basic fibroblast growth factor (FGF-2), hepatocyte growth factor (HGF; all from Santa Cruz Biotechnology); brain-derived neurotrophic factor (BDNF), and neurotrophin-3 (NT-3; both from Chemicon, Harrow, UK).

\section{Statistical analysis}

Data were analyzed using Student's $t$ test to compare paired results and one-way ANOVA with post hoc testing for comparison of multiple sets. 
Values expressed are the mean $\pm \mathrm{SE}$ of the mean from at least three independent experiments, unless otherwise stated.

\section{Results}

\section{Characterization of cultures}

To examine the survival effects of oligodendrocyte-derived signals, E16 cortical neurons were cultured under serum-free B27 supplemented conditions. Neurons (identified by $\beta$-tubulin III staining) represented $95.5 \pm 1.3 \%(n=3)$ of the cells $24 \mathrm{hr}$ after plating, and $94.6 \pm 1.6 \%(n=3)$ of the cells $5 \mathrm{~d}$ after plating. The remainder was predominantly astrocytes, with $0.4 \% \mathrm{O} 4$-positive and $0.2 \%$ GalC-positive cells. Insulin-free medium (DMEM/ Sato) was used to condition (a) enriched OPC cultures $4 \mathrm{hr}$ after withdrawal of B104 conditioned medium (CM) [oligodendrocyte-conditioned media (OCM) day 1 (OCM1)] and (b) enriched OPC cultures after differentiation in B27 supplemented DMEM for 96 hr [OCM day 4 (OCM4)]. Four hours after withdrawal of B104CM, $85.7 \pm 2.9 \%$ of cells were A2B5 positive, $7.8 \pm 1.3 \%$ were GalC positive, and $4.2 \pm 1.6 \%$ were GFAP positive. No cells of neuronal ( $\beta$-tubulin III staining) or microglial phenotype (lectin from Bandiera simplicifolia BS-1 staining) were detected. $96 \mathrm{hr}$ after culture in DMEM/2\% B27, $12.6 \pm 7.8 \%$ of cells were A2B5 positive, $78.8 \pm 3.7 \%$ were GalC positive and $4.3 \pm 3.2 \%$ were GFAP positive. Thus, OCM1 represents media conditioned predominantly by OPCs, and OCM4 represents media conditioned predominantly by differentiated oligodendrocytes.

\section{Oligodendrocyte-conditioned media increase survival of neurons}

We have shown previously that OCM1 and OCM4, which were shown to contain comparable levels of IGF-1, increase the survival of E16 cortical neurons plated for $24 \mathrm{hr}$ before exposure to test conditions (Wilkins et al., 2001). We therefore wished to extend these observations to address the effect of OCM1 and OCM4 on different maturational stages of neurons. Preliminary observations on the survival of these cultures led us to adopt two plating densities for the experiment. Neurons were plated at $1.1 \times 10^{3}$ cells per $\mathrm{mm}^{2}$ for $24 \mathrm{hr}$ ("young"), or $1.4 \times 10^{3}$ cells per $\mathrm{mm}^{2}$ for $120 \mathrm{hr}$ ("aged") before exposure to conditioned media. OCM1 and OCM4 increased the survival of young and aged neurons as measured by the number of $\beta$-tubulin-positive cells per field $72 \mathrm{hr}$ after exposure. The effect of OCM4 was significantly greater than OCM1 in young neurons (Fig. $1 A$ ). In contrast, OCM1 and OCM4 had comparable effects on survival of aged neurons (exposed after $120 \mathrm{hr}$ ) compared with nonconditioned media (Fig. 1B). OCM1- and OCM4-mediated survival depended on suppression of apoptosis as shown by morphological analysis using Hoechst and TUNEL staining (Fig. 1C). Blockade of IGF-1 resulted in a significant reduction in aged neuronal survival, consistent with previous findings implicating oligodendrocyte-derived IGF-1 as an important mediator of neuronal survival (Fig. 1 B) (Wilkins et al., 2001).

\section{Oligodendrocyte-mediated survival is dependent on the PI3kinase-Akt-signaling pathway}

To examine the intracellular signaling pathways that mediate OCM-derived neuronal survival, the effect of specific inhibitors of PI3 kinase and MAP kinase pathways were examined. Addition of the PI3 kinase inhibitors LY294002 (10 $\mu \mathrm{M})$ and wortmannin (100 nM; data not shown) significantly reduced the prosurvival effect of both OCM1 and OCM4 (Fig. 2A; data shown for OCM4), whereas the addition of the MAPK/Erk pathway inhibitor PD98059 (30 $\mu \mathrm{M})$ had no effect on cell survival. One of the
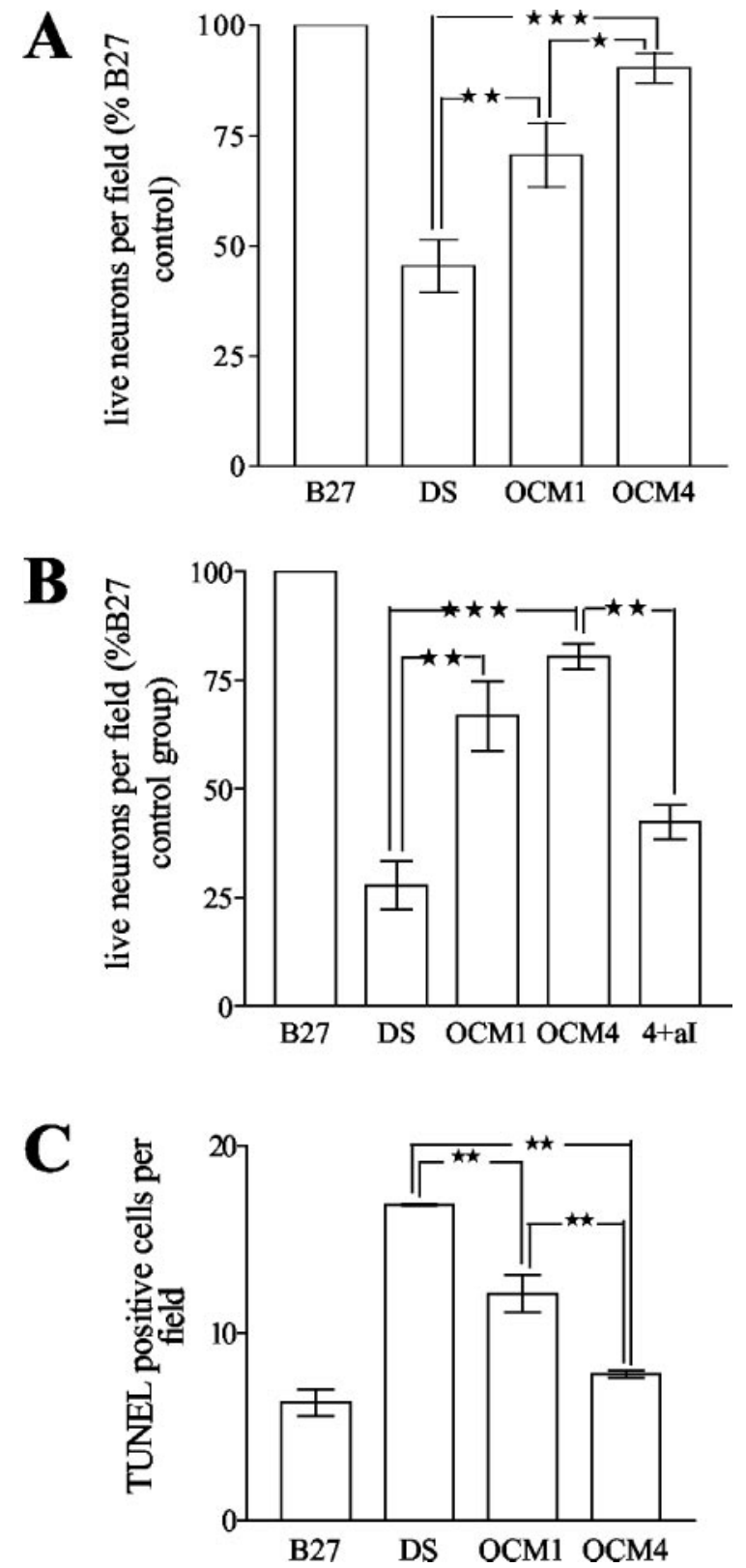

Figure 1. A, Oligodendrocyte-conditioned media increase the survival of young neurons. Effect of nonconditioned medium (DS), medium conditioned by freshly plated OPCS (OCM1), and medium conditioned by predominantly GalC-positive oligodendrocytes (OCM4) on numbers of live ( $\beta$-tubulin-positive) neurons is shown. Neurons were cultured for $24 \mathrm{hr}$ and exposed to test conditions for $72 \mathrm{hr}\left(n=5 ;{ }^{*} p<0.05 ;{ }^{* *} p<0.01\right.$; $\left.^{* * *} p<0.001\right)$. B, Oligodendrocyteconditioned media increase the survival of aged neurons. Effect of nonconditioned medium (DS), OCM1, OCM4, and OCM4 plus neutralizing antibodies to IGF-1 $(20 \mu \mathrm{g} / \mathrm{ml} ; 4+$ al) on numbers of live ( $\beta$-tubulin-positive) neurons is shown. Neurons were cultured for $120 \mathrm{hr}$ and exposed to test conditions for $72 \mathrm{hr}\left(n=4\right.$; $\left.{ }^{* *} p<0.01 ;{ }^{* * *} p<0.001\right)$. C, Oligodendrocyteconditioned media decrease apoptosis of young neurons. Effect of nonconditioned medium (DS), OCM1, and OCM4 on numbers of TUNEL-positive neurons is shown. Neurons were cultured for $24 \mathrm{hr}$ and exposed to test conditions for $72 \mathrm{hr}\left(n=3\right.$; $\left.{ }^{* *} p<0.01\right)$.

principal targets of PI3 kinase activation is Akt (protein kinase B). We therefore studied Akt phosphorylation in neurons exposed to OCM. Neurons were cultured for 24 or $120 \mathrm{hr}$ before washing with DMEM and then exposed to OCM or nonconditioned media. IGF-1 (100 ng/ml), which is known to activate the Akt phosphorylation pathway, was used as a positive control. Cultures were exposed to test condition for $30 \mathrm{~min}$ before lysis and immu- 

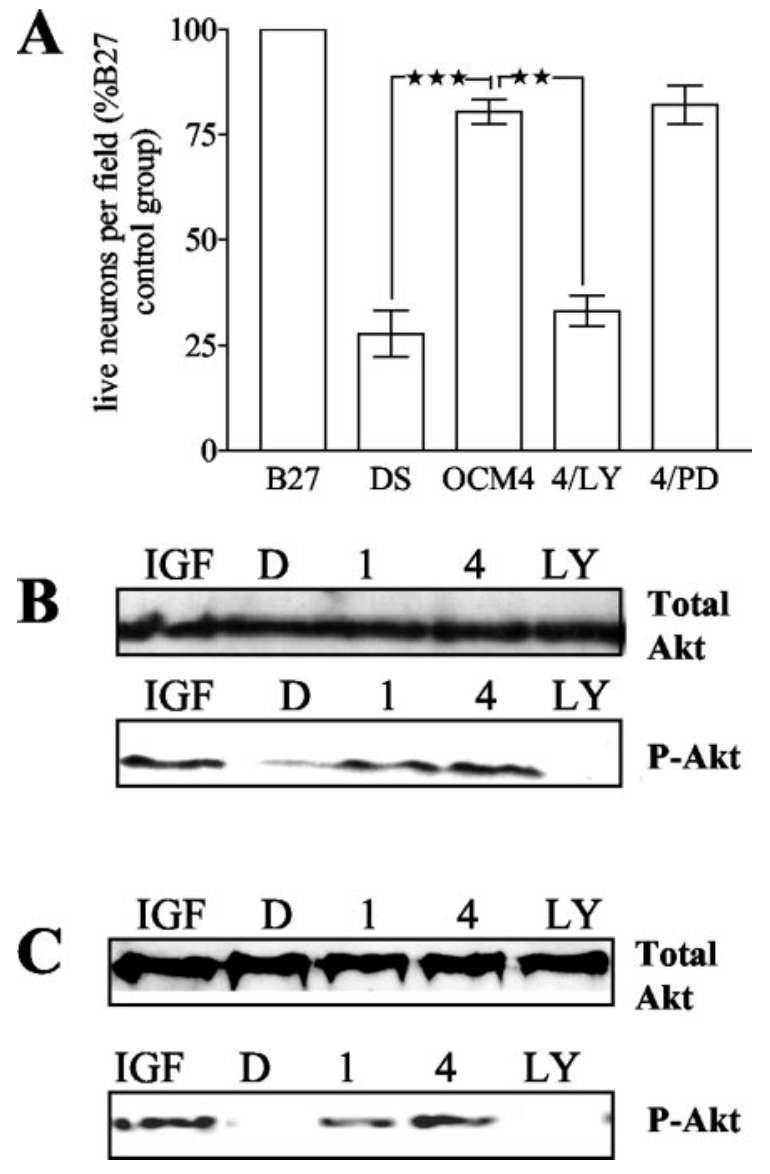

Figure 2. A, PI3kinase inhibitor, but not MAPK/Erk pathway inhibitor, abrogates the $0 \mathrm{CM} 4$ survival effects on neurons. Effect of nonconditioned medium (DS), OCM4, OCM4 plus LY294002 (10 $\mu \mathrm{m} ; 4 / \mathrm{LY}$ ), and OCM4 plus PD98059 (30 $\mu \mathrm{m}$; 4/PD) on numbers of live ( $\beta$-tubulin-positive) neurons is shown. Neurons were cultured for $120 \mathrm{hr}$ and exposed to test conditions for $72 \mathrm{hr}$ $\left(n=4 ;{ }^{* *} p<0.01 ;{ }^{* *} p<0.001\right) . B$, C, Oligodendrocyte-conditioned media increase Akt phosphorylation in neurons. $B$, Akt phosphorylation in young neurons (cultured for $24 \mathrm{hr}$ ) exposed to IGF-1 (100 ng/ml; IGF), nonconditioned media (D), OCM1 (1), OCM4 (4), or 0CM4 plus LY294002 (10 $\mu \mathrm{m}$; LY) for $30 \mathrm{~min}$. Bottom, Serine473-phospho-Akt immunoblot. Top, Total-Akt immunoblot of the same membrane. Representative blot of four independent experiments. $C$, Akt phosphorylation in aged neurons (cultured for $120 \mathrm{hr}$ ) and exposed to IGF-1 (100 ng/ml; IGF), nonconditioned media (D), OCM1 (1), OCM4 (4), or OCM4 plus LY294002 (10 $\mu$ m; LY) for 30 min. Bottom, Serine473-phospho-Akt immunoblot. Top, Total-Akt immunoblot of the same membrane. Representative blot of three independent experiments.

noblotting with an antibody specific for the phosphorylated form of Akt (P-Akt; at serine 473). Both young and aged neurons cultured in nonconditioned media showed small amounts of P-Akt, whereas neurons exposed to both OCM1 and OCM4 showed substantially increased levels of P-Akt relative to control (Fig. $2 B, C)$. The PI3 kinase inhibitor LY294002 (10 $\mu \mathrm{M})$ reduced P-Akt levels to undetectable levels. These results show that the PI3 kinase-Akt pathway is sufficient and necessary for OCMmediated survival.

OCM4 increases the length of phosphorylated neurofilament per neuron via MAP kinase/Erk1/2 pathways

In addition to effects on survival, we examined the influence of OCM on the morphology of cultured neurons and specifically on neurofilament phosphorylation. Neurons were cultured at $1.4 \times$ $10^{3}$ cells per $\mathrm{mm}^{2}$ for $120 \mathrm{hr}$ before changing to nonconditioned media, OCM1, OCM4, or fresh DMEM/2\% B27 as control. After an additional $72 \mathrm{hr}$, cultures were fixed and stained for $\beta$-tubulin or SMI312, and an analysis of process lengths was undertaken. To correct for the differences in cell survival observed under the varied conditions, lengths of processes staining for $\beta$-tubulin or SMI312 were divided by the average number of surviving cells per field, thus providing a quantitative measure of process length per live cell. Initial studies using $\beta$-tubulin staining, which labels all neuronal processes, showed increased neurite lengths in cells cultured in the presence of OCM4 (data not shown), but this difference was not statistically significant. In view of the fact that oligodendrocytes normally ensheath only axons (Lubetzki et al., 1993), we attempted next to examine the specific effects of OCM on axons. Neurofilament phosphorylation, as identified by the antibody SMI312, which is specific for phospho-epitopes in neurofilaments, was examined as a marker of axonal integrity and length. Preliminary experiments showed that SMI312 expression occurred after $\sim 96 \mathrm{hr}$ in culture, and that SMI312 staining patterns were distinct from $\beta$-tubulin III staining, an observation compatible with previous reports suggesting that SMI312 staining is axon-specific (Lee et al., 1987) (Fig. 3A,B). OCM4 alone showed a significant difference from the control, with SMI312 length increased by $\sim 50 \%$ per cell, but interestingly OCM1 showed no effect (Fig. 3C-F).

In view of previous reports suggesting the importance of MAPkinase for neuronal differentiation and neurite growth, we next examined the role of this pathway in OCM-mediated enhancement of axonal length. First, we analyzed whether MAPkinase was activated in neurons by OCM. Compared with immunoblots of lysates from aged neurons exposed to nonconditioned media for $30 \mathrm{~min}$, neurons exposed to OCM4 had increased levels of P-Erk1/2 (Fig. 4A). The MAPK/Erk pathway inhibitor PD98059 $(30 \mu \mathrm{M})$ added to OCM4 reduced P-Erk1/2 levels to control levels.

Next, we investigated effects of the MAPK/Erk pathway inhibitor PD98059 $(30 \mu \mathrm{M})$ on SMI312 staining. The addition of PD98059 to OCM4 did not influence cell numbers (Fig. 2A) but significantly reduced SMI312 lengths per cell to control levels compared with OCM4 alone (Figs. $4 B$ ). Together, these results demonstrate that factors released by differentiated oligodendrocytes increase axonal phosphorylated neurofilament levels through a MAPkinase-dependent mechanism.

\section{Differentiated oligodendrocytes secrete GDNF}

The differential effect of OCM1 and OCM4 on axon neurofilament phosphorylation levels suggests that OCM4 contains additional factor(s) compared with OCM1. In addition, we have previously reported comparable levels of IGF-1 within OCM1 and OCM4. Therefore studies were undertaken to characterize further the composition of conditioned media. Nonconditioned media, OCM1 and OCM4, together with type-1 ACM (acting as a positive control for certain factors) were concentrated and electrophoresed by SDS-PAGE. Resulting gels were transferred to membranes and probed with antibodies against a series of growth factors. These included IGF-2, NGF- $\beta$, BDNF, NT-3, CNTF, GDNF, FGF-2, and HGF. GDNF was detected in OCM4 and ACM (Fig. 5A). The GDNF found in OCM4 was not thought to be attributable to contaminating astrocytes because both OCM1 and OCM4 are derived from cultures containing similar percentages of astrocytes (4.2 and 4.3\%, respectively). Examination of GDNF in medium conditioned by purified astrocytes at $1 \mathrm{~d}$ in vitro (DIV) and 4 DIV (corresponding to OCM1 and OCM4) revealed comparable levels of GDNF (Fig. 5B). Furthermore, immunostaining revealed positive GDNF expression in differentiated oligodendrocytes (4 DIV) and not in OPCs (1 DIV) (Fig. 

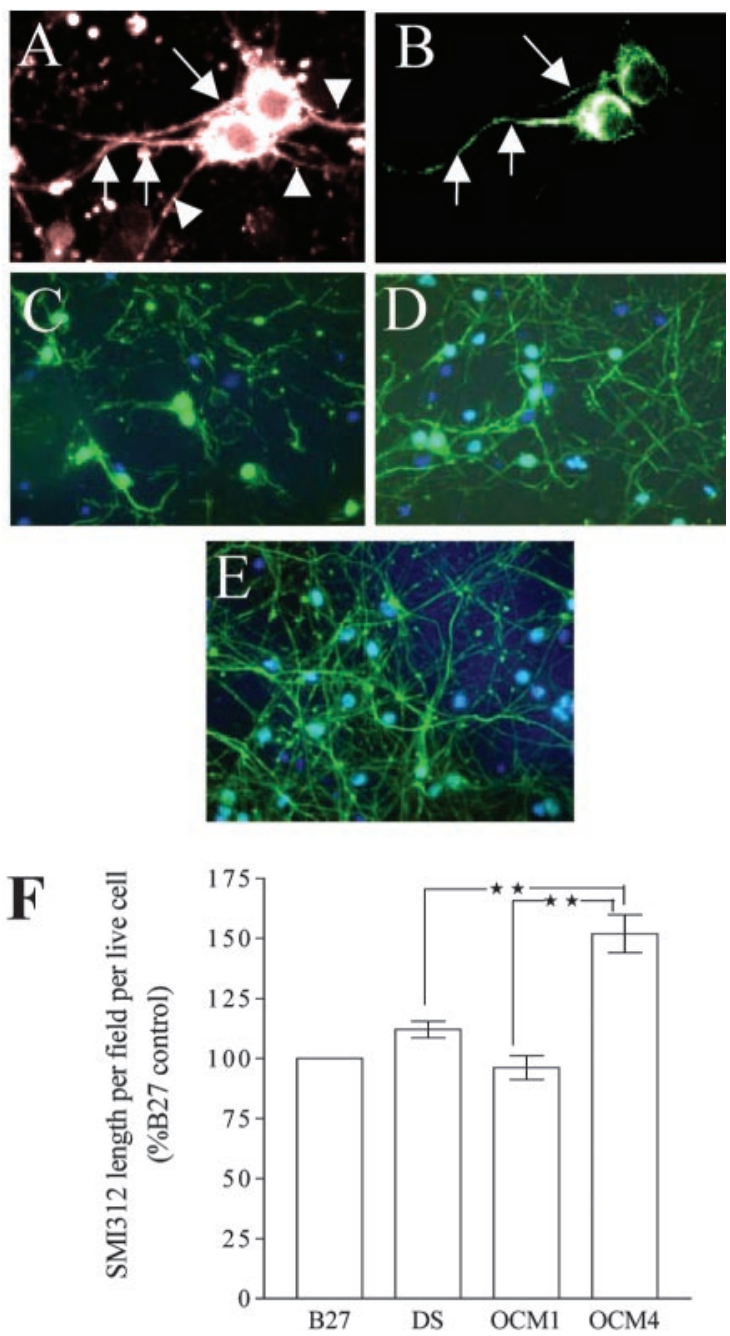

Figure 3. OCM4 increases SMI312-positive process length per cell in aged neurons. Immunographs show neuronal cultures (8 DIV) stained for $\beta$-tubulin (3A) and SMI312 (3B), showing axons (arrows) and other neurites (arrowheads). $(-E$, Effect of nonconditioned medium $(C)$ medium conditioned by freshly plated OPCS (OCM1) (D), and medium conditioned by predominantly GalC-positive oligodendrocytes (OCM4) on SMI312 (phosphorylated neurofilament; green) staining in aged neuronal cultures ( $E$ ). Nuclei are stained with Hoechst (blue). F, Effect of nonconditioned medium (DS), $0 C M 1$, and $0 C M 4$ on length of SMI312-stained processes per live neuron in aged neurons (cultured for $120 \mathrm{hr}$ and exposed to test conditions for $72 \mathrm{hr}$; expressed as a percentage of values obtained from culturing neurons in B27 throughout) $\left(n=4\right.$; ${ }^{* *} p<$ $0.01)$.

$5 C)$. Together these data are consistent with the production of GDNF by differentiated oligodendrocytes. No other factors were detected in OCM1 or OCM4 by this method.

To elucidate the biological relevance of OCM4-derived GDNF, E16 cortical neurons were cultured as described previously for 5 DIV (aged) before exposure to OCM4 or OCM4 plus neutralizing antibodies to GDNF. Anti-GDNF antibodies had no significant effect on OCM4-induced neuronal survival (OCM4, $74.6 \pm 4.6 \%$; OCM4 plus anti-GDNF, $70.4 \pm 7.9 \%)$. After an additional 3 DIV, cultures were analyzed for SMI312 length staining per neuron. The increase in SMI312 length per neuron seen with OCM4 was significantly reduced by the addition of neutralizing antibodies to GDNF (Fig. 5D-F). Finally, GDNF was shown to activate the MAPK/Erk pathway (Fig. 5G), consistent with the observation that this pathway mediates axonal-specific effects, and providing additional evidence that GDNF is an active constituent of OCM4
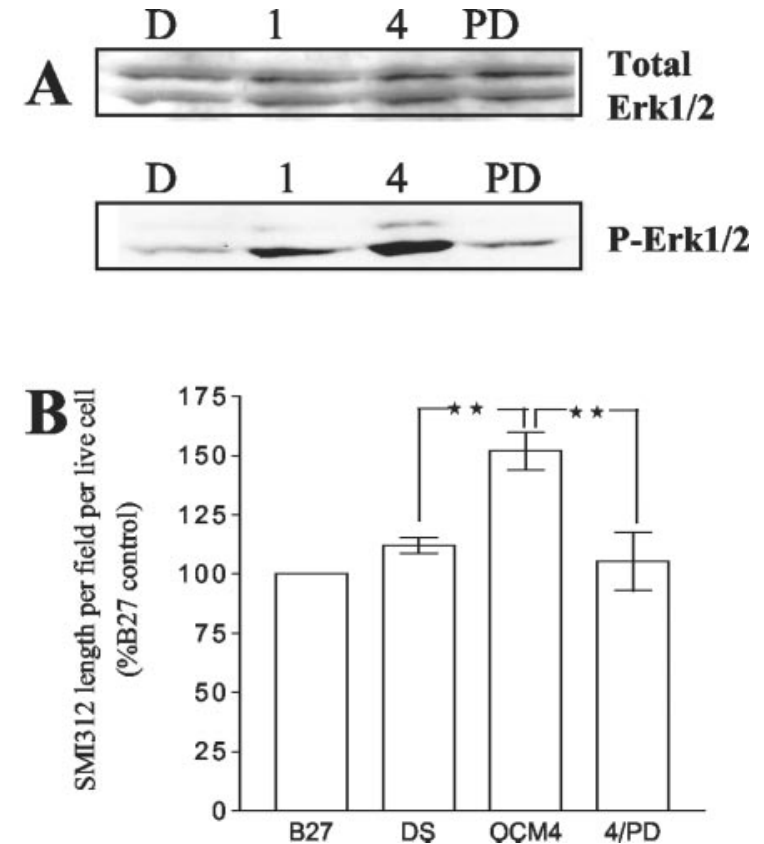

Figure 4. A, Oligodendrocyte conditioned media increase MAPK/Erk1/2 phosphorylation in aged neurons. MAPK/Erk $1 / 2$ phosphorylation in aged neurons (cultured for $120 \mathrm{hr}$ ) and exposed to nonconditioned media (D), OCM1 (1), OCM4 (4), and OCM4 plus PD98059 (30 $\mu \mathrm{m}$; PD) for 30 min is shown. Bottom, Phospho-Erk1/2 immunoblot. Top, Total-Erk1/2 immunoblot of the same membrane. Representative blot of three independent experiments. $B$, Effect of nonconditioned medium (DS), 0CM4, and OCM4 plus PD98059 (4/PD; $30 \mu \mathrm{M}$ ) on the length of SMI312stained processes per live neuron in aged neurons (cultured for $120 \mathrm{hr}$ and exposed to test conditions for $72 \mathrm{hr}$; expressed as a percentage of values obtained from culturing neurons in B27 throughout) $\left(n=4 ;{ }^{* *} p<0.01\right)$.

\section{Discussion}

In this study, in examining oligodendrocyte-neuronal interactions, we show that oligodendrocytes and OPCs release factors that promote the survival of cortical neurons and influence levels of phosphorylated neurofilaments within axons by distinct signaling pathways. The former process occurs through the PI3kinase/Akt-signaling pathway and the latter by activation of MAPK/Erk1/2. We provide several lines of evidence that GDNF production by differentiated oligodendrocytes contributes significantly to increases in axonal phosphorylated neurofilament levels, a process independent of cell survival.

\section{Oligodendrocyte-derived factors promote neuronal survival via the PI3kinase/Akt pathway}

Schwann cells in the peripheral nervous system and astrocytes in the CNS produce molecules that are trophic for neurons (Banker, 1980; Ohgoh et al., 1988; Riethmacher et al., 1997). However, less attention has been focused on oligodendrocytes as a source of trophic factors. Oligodendrocytes are known to influence the development of axons and neurons; for example, they regulate axon size and caliber, and ultrastructural maturation is induced by oligodendrocyte ensheathment, consistent with the interpretation that myelinated axons require continual local oligodendrocyte contact during development (Colello et al., 1994; Sanchez et al., 1996; Brady et al., 1999). These processes rely on oligodendrocyte contact with the axon, but soluble factors produced by oligodendrocytes also appear to play an important role in promoting and modifying neuronal function (Du and Dreyfus, 2002). Oligodendrocyte-derived soluble factors induce sodium channel clustering along axons (Kaplan et al., 1997). Byravan et 
al. (1994) also showed that factors derived from oligodendrocyte cell lines increase neurite extension in PC12 cells. In terms of direct survival effects, Meyer-Franke et al. (1995) detected an as yet uncharacterized soluble protein of $>30 \mathrm{kDa}$ derived from mature oligodendrocytes that improved the survival of postnatal retinal ganglion cells. We have shown that oligodendrocytes and OPCs secrete factors, including IGF-1, which increase the survival of young and aged cortical neurons (Wilkins et al., 2001; our observations). In the present study, we examined signaling mechanisms that underlie the prosurvival effect of oligodendrocyte-conditioned medium derived from both OPCs and differentiated oligodendrocytes and extend previous observations to assess these effects on older neurons in culture. We show that OCM increases phosphorylation of Akt within neurons compared with nonconditioned medium and that the PI3kinase inhibitor LY294002 significantly reduces neuronal survival.

The PI3kinase/Akt pathway is a critical cellular survival pathway (Kaplan and Miller, 2000). Akt pathways are particularly important in mediating neuronal survival, for instance, in determining the response of sympathetic neurons to depolarization and NGF exposure (Crowder and Freeman, 1998; Vaillant et al., 1999). These reports highlight the convergence of anti-apoptotic processes on Akt because both exposure to $\mathrm{KCl}$, leading to depolarization of the cell, and NGF treatment of cells leads to Akt phosphorylation and a reduction in apoptosis. Our findings are indirectly supported by the report of Dudek et al. (1997) showing that IGF-1 mediates prosurvival effects on cerebellar neurons via PI3kinase/Akt pathways. The PI3kinase/Akt pathway has also been implicated in the survival of other neuronal types such as sensory neurons, cerebellar granule cells, and retinal ganglion cells (Klesse and Parada, 1998; Diem et al., 2001; Encinas et al., 2001).

The survival effect of OCM1 (medium conditioned by cultures that are predominantly OPCs) on young neurons is significantly less compared with OCM4 (medium conditioned by cultures that are predominantly differentiated oligodendrocytes), implying differences in the trophic potential of oligodendrocytes as they differentiate. This trend is seen in the aged neurons but does not reach significance, which may point toward different trophic requirements of cells as they mature. It is well established that CNS neurons, in contrast to peripheral neurons, require multiple signals for optimal survival and maturation, although the precise identity and combination of factors may differ depending on neuronal type and age (for review, see Goldberg and Barres, 2000).

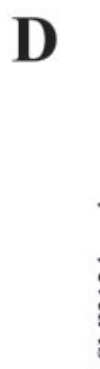

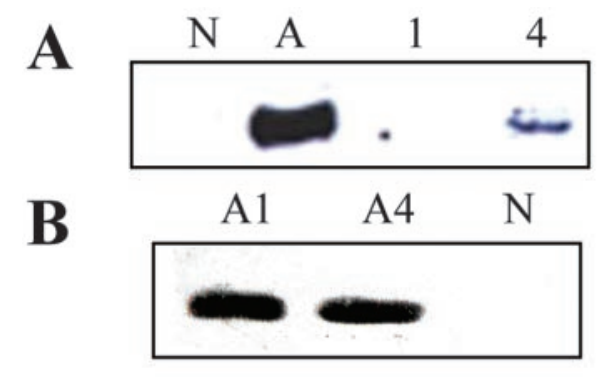
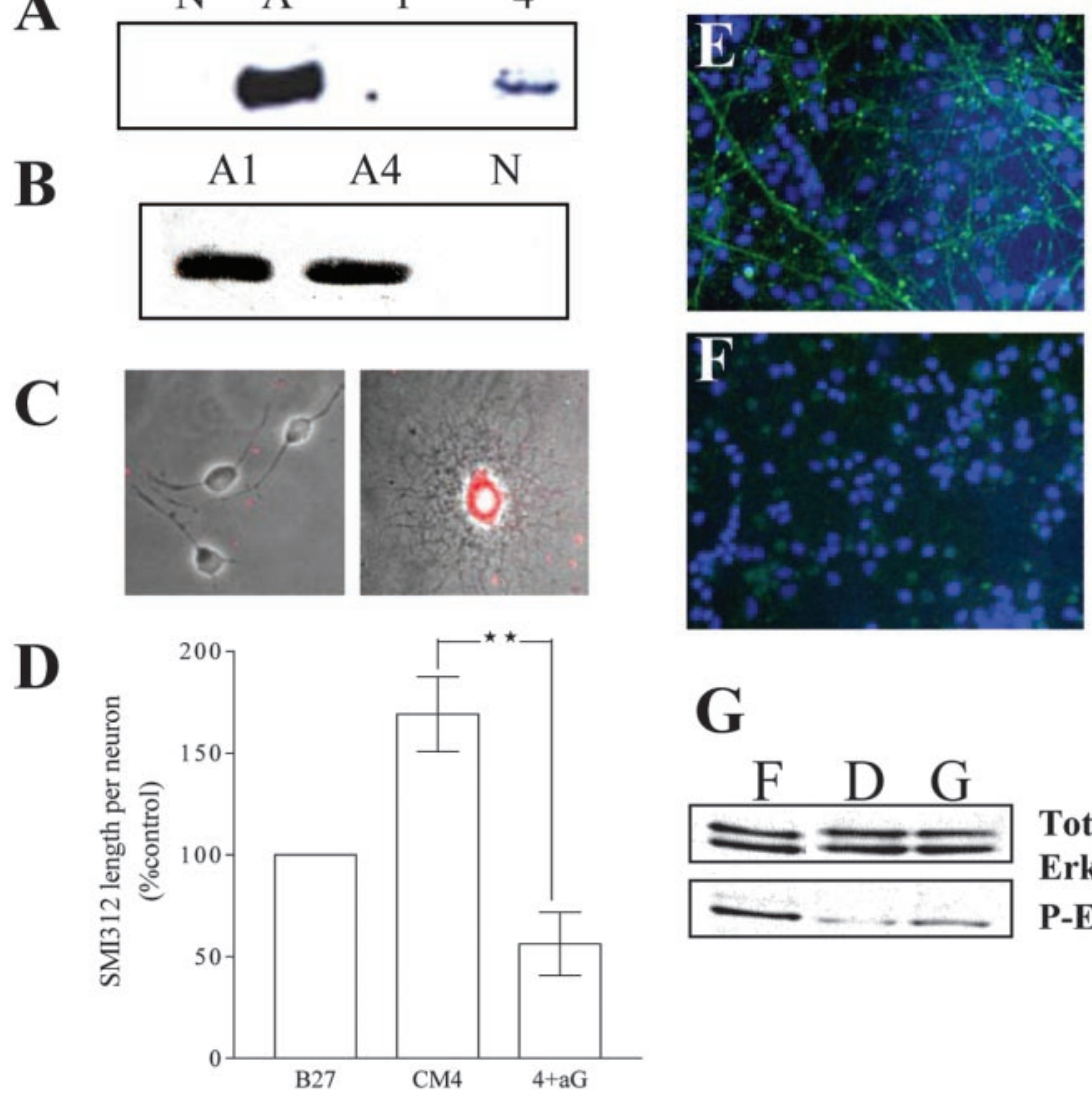

Total

Erk 1/2

P-Erk

Figure 5. A, Oligodendrocyte-conditioned medium contains GDNF. Immunoblots of nonconditioned medium $(N)$ are shown astrocyte-conditioned medium ( $A$ ), OCM1 (1), and OCM4 (4) are blotted for antibodies against GDNF (representative blots of three independent experiments). $B$, GDNF production by astrocytes is not influenced by the maturation state of astrocytes. Immunoblots GDNF and showing comparable levels of GDNF are shown (equal amounts of protein loaded per lane; see Materials and Methods; representative blots of three independent experiments). C, GDNF staining within a differentiated oligodendrocyte. Immunocytoshown with superimposed rhodamine-labeled GDNF staining of the same field, showing that differentiated oligodendrocytes presses per live neuron in aged neurons (cultured for $120 \mathrm{hr}$ and exposed to test conditions for $72 \mathrm{hr}$; expressed as a percentage 作 staining in aged neuronal cultures. Nuclei stained with Hoechst are shown (blue). G, GDNF increases MAPK/Erk1/2 phosexposed to DMEM/10\% fetal calf serum $(F)$, nonconditioned media $(D)$, and nonconditioned media plus recombinant GDNF (10 (representative immunoblot of four independent experiments).

Oligodendrocyte-derived soluble factors increase axon length through the MAPK/Erk1/2 signaling pathway

In addition to demonstrating a prosurvival effect of OCM on neurons, we next asked whether oligodendrocyte-derived soluble factors could influence neuronal morphology. Initial studies examining $\beta$-tubulin-positive processes revealed a nonsignificant trend showing increased process formation. However, $\beta$-tubulin is not specific for axons and also identifies dendrites. In view of the fact that oligodendrocytes ensheath axons and not dendrites (Lubetzki et al., 1993), we examined the effect of OCM on SMI312-positive neuronal process formation. SMI312 identifies phosphorylated neurofilaments and has previously been shown to be axon specific, an observation that correlates with our findings when cultures were costained for $\beta$-tubulin and SMI312 (Lee 
et al., 1987; Lubetzki et al., 1993). Phosphorylation of neurofilaments during development is fundamental to maturation and stabilization of the neuronal cytoskeleton, as well as an increase in axonal diameter (Carden et al., 1987; Sanchez et al., 2000). We show that OCM4 significantly increases SMI312 positive process formation per surviving neuron, whereas OCM1 has no effect. This result is consistent with a role for the differentiated oligodendrocyte and not the oligodendrocyte precursor toward formation of the neuron-oligodendrocyte-myelinated unit. Mechanisms by which neurofilaments become phosphorylated are complex, but a role for MAPK/Erk has been established (Pang et al., 1995; Veeranna et al., 1998; Li et al., 1999). We found that OCM4 increases phosphorylation of MAPK/Erk1/2 in neurons and that the MAPK/Erk pathway inhibitor PD98059 significantly reduces the effect of OCM4 to control levels. Importantly, MAPK inhibition has no effect on neuronal survival. Together, these results suggest that OCM4, via MAPK/Erk, may promote axonal survival or facilitate phosphorylation of axonal neurofilaments. In vivo support for our observations is provided by the study of myelin mutant animals, in which impaired oligodendrocyte maturation is associated with neurofilament hypophosphorylation (Sanchez et al., 2000). The effects on neuronal survival and axon structure raise the possibility that axons and neuronal cell bodies have distinct survival requirements. A recent study demonstrating that axon degeneration occurs by a different mechanism from that of neuronal cell body apoptosis supports such an idea (Finn et al., 2001; Raff et al., 2002).

\section{Differentiated oligodendrocytes secrete GDNF}

The observation that only OCM4 influenced axonal-phosphorylated neurofilament levels led to the identification of GDNF within OCM4 and not OCM1. Crucially, the demonstration that the addition of anti-GDNF antibodies to OCM4 attenuates its effects on axonal-phosphorylated neurofilament levels along with the finding that GDNF activates MAPkinase/Erk implicates oligodendrocyte-derived GDNF in these axon-specific effects.

GDNF was initially identified as a potent survival factor for midbrain dopaminergic neurons (Lin et al., 1993). It is related to the transforming growth factor- $\beta$ superfamily of molecules, and more recently, additional members of the GDNF family have been identified: neurturin (NTN), persephin (PSP), and artemin, with each factor mediating its signal via a common receptor tyrosine kinase (Ret) and ligand specificity dictated by a specific GDNF family receptor- $\alpha$ (Saarma and Sariola, 1999). In addition to the effects on dopaminergic neurons, the growth factor effects of GDNF family members have been extended to motor neurons, enteric neurons, and the process of ureteric budding during kidney morphogenesis (Goyal and Hirano, 1996; Arce et al., 1998; Saarma and Sariola, 1999). Expression of GDNF family members and their receptors has also been noted in other parts of the CNS including cortical neurons and astrocytes, suggesting the potential importance of the molecule during acute brain injury (Nicole et al., 2001).

Our report of GDNF production by oligodendrocytes is consistent with previous identification of GDNF family member production by cells of oligodendrocyte lineage (Strelau and Unsicker, 1999). Cell lines derived from oligodendrocyte precursors (OLI-neu) and lines derived from immature oligodendrocytes (OLN-93) expressed GDNF mRNA, along with mRNA from other members of the GDNF family (NTN and PSP). Expression of NTN and PSP mRNA was greatly increased in the OLN-93 line compared with the OLI-neu. In addition, examination of pri- mary OPC and differentiated oligodendrocyte cultures detected GDNF mRNA in differentiated cells, in keeping with the present study.

Previous studies have shown GDNF activation of MAPK/Erk, whereas others have demonstrated the importance of this pathway in the process of neurofilament phosphorylation (Veeranna et al., 1998; Nicole et al., 2001). The discovery of a role for GDNF in neurofilament phosphorylation, independent of cell survival, is novel and provides additional insights into the role of this growth factor.

Many neurotrophic factors have been identified that activate several intracellular pathways in vitro, and the requirement for combinations of factors is apparent for CNS neurons with the precise nature of such signals being dependent on type, region, and age of neurons (Snider, 1994; Meyer-Franke et al., 1995; Goldberg and Barres, 2000). We identified GDNF production by differentiated oligodendrocytes in addition to the previous finding of IGF-1 production by both OPCs and differentiated oligodendrocytes.

The demonstration that oligodendrocyte-derived soluble factors have disparate effects on cortical neurons through activation of distinct intracellular signaling pathways is of considerable interest for diseases such as multiple sclerosis. In addition to the destruction of the oligodendrocyte myelin unit, neuronal loss and axonal injury is well recognized in multiple sclerosis (Trapp et al., 1998). Loss of oligodendrocyte-derived signals in chronically demyelinated lesions may contribute to the chronic axonopathy of multiple sclerosis. Although this study and others addressing oligodendrocyte neuronal interactions have focused on developmental systems, there is increasing evidence that neurons of the mature CNS require continual trophic support (Acheson et al., 1995). The study of myelin mutants has shown that various components of the myelin membrane appear important in maintaining axonal integrity. For instance, mice lacking proteolipid protein develop a delayed-onset axonopathy with a slow and progressive loss of nerve fibers (Griffiths et al., 1998). The continued attrition of axons seen in chronically demyelinated multiple sclerosis lesions, in the absence of acute inflammation, also supports the hypothesis that continued oligodendrocyte-derived signals are necessary to maintain neuronal integrity (Coles et al., 1999; Kornek et al., 2000).

\section{References}

Acheson A, Conover JC, Fandl JP, DeChiara TM, Russell M, Thadani A, Squinto SP, Yancopoulos GD, Lindsay RM (1995) A BDNF autocrine loop in adult sensory neurons prevents cell death. Nature 374:450-453.

Arce V, Pollock RA, Philippe JM, Pennica D, Henderson CE, deLapeyriere O (1998) Synergistic effects of schwann- and muscle-derived factors on motoneuron survival involve GDNF and cardiotrophin-1 (CT-1). J Neurosci 18:1440-1448.

Atwal JK, Massie B, Miller FD, Kaplan DR (2000) The TrkB-Shc site signals neuronal survival and local axon growth via MAPK/Erk pathway and PI3-kinase. Neuron 27:265-277.

Banker GA (1980) Trophic interactions between astroglial cells and hippocampal neurons in culture. Science 209:809-810.

Brady ST, Witt AS, Kirkpatrick LL, de Waegh SM, Readhead C, Tu PH, Lee VM (1999) Formation of compact myelin is required for the maturation of the axonal cytoskeleton. J Neurosci 19:7278-7288.

Byravan S, Foster LM, Phan T, Verity AN, Campagnoni AT (1994) Murine oligodendroglial cells express nerve growth factor. Proc Natl Acad Sci USA 91:8812-8816.

Cannoll PD, Musacchio JM, Hardy R, Reynolds R, Marchionni MA, Salzer JL (1996) GGF/neuregulin is a neuronal signal that promotes the proliferation and survival and inhibits the differentiation of oligodendrocyte precursors. Neuron 17:229-243. 
Carden MJ, Trojanowski JQ, Schlaepfer WW, Lee VM (1987) Two-stage expression of neurofilament polypeptides during rat neurogenesis with early establishment of adult phosphorylation patterns. J Neurosci 7:3489-3504

Colello RJ, Pott U, Schwab ME (1994) The role of oligodendrocytes and myelin on axon maturation in the developing rat retinofugal pathway. J Neurosci 14:2594-2605.

Coles AJ, Wing MG, Molyneux P, Paolillo A, Davie CM, Hale G, Miller D, Waldmann H, Compston A (1999) Monoclonal antibody treatment exposes three mechanisms underlying the clinical course of multiple sclerosis. Ann Neurol 46:296-304.

Crowder RJ, Freeman RS (1998) Phosphatidylinositol-3 kinase and Akt kinase are necessary and sufficient for the survival of nerve growth factordependent sympathetic neurons. J Neurosci 18:2933-2943.

Diem R, Meyer R, Weishaupt JR, Bahr M (2001) Reduction of potassium currents and phosphatidylinositol 3-kinase-dependent AKT phosphorylation by tumor necrosis factor-(alpha) rescues axotomized retinal ganglion cells from retrograde cell death in vivo. J Neurosci 21:2058-2066.

Du Y, Dreyfus CF (2002) Oligodendrocytes as providers of growth factors. J Neurosci Res 68:647-654.

Dudek H, Datta SR, Franke TF, Birnbaum MJ, Yao R, Cooper GM, Segal RA, Kaplan DR, Greenberg ME (1997) Regulation of neuronal survival by the serine-threonine protein kinase Akt. Science 275:661-665.

Encinas M, Tansey MG, Tsui-Pierchala BA, Comella JX, Milbrandt J, Johnson Jr EM (2001) c-src is required for glial cell line derived neurotrophic factor (GDNF) family ligand-mediated neuronal survival via a phopshatidylinositol-3 kinase (PI-3K)-dependent pathway. J Neurosci 21:1464-1472.

Finn JT, Weil M, Archer F, Siman R, Srinivasan A, Raff MC (2000) Evidence that Wallerian degeneration and localized axon degeneration induced by local neurotrophin deprivation do not involve caspases. J Neurosci 20: 1333-1341.

Goldberg JL, Barres BA (2000) The relationship between neuronal survival and regeneration. Annu Rev Neurosci 23:579-612.

Goyal RK, Hirano I (1996) The enteric nervous system. N Engl J Med 334:1106-1115.

Griffiths I, Klugmann M, Anderson T, Yool D, Thomson C, Schwab MH, Schneider A, Zimmermann F, McCulloch M, Nadon N, Nave KA (1998) Axonal swellings and degeneration in mice lacking the major proteolipid of myelin. Science 280:1610-1613.

Henderson CE (1996) Role of neurotrophic factors in neuronal development. Curr Opin Neurobiol 6:64-70.

Kaplan DR, Miller FD (2000) Neurotrophin signal transduction in the nervous system. Curr Opin Neurobiol 10:381-391.

Kaplan MR, Meyer-Franke A, Lambert S, Bennett V, Duncan ID, Levinson SR, Barres BA (1997) Induction of sodium channel clustering by oligodendrocytes. Nature 386:724-728.

Klesse LJ, Parada LF (1998) p21 ras and phosphatidylinositol-3 kinase are required for survival of wild-type and NF1 mutant sensory neurons. J Neurosci 18:10420-10428.

Kornek B, Storch MK, Weissert R, Wallstroem E, Stefferl A, Olsson T, Linington C, Schmidbauer M, Lassmann H (2000) Multiple sclerosis and chronic autoimmune encephalomyelitis: a comparative quantitative study of axonal injury in active, inactive and remyelinated lesions. Am J Pathol 157:267-276.

Lee VM, Carden MJ, Schlaepfer WW, Trojanowski JQ (1987) Monoclonal antibodies distinguish several differentially phosphorylated states of the two largest rat neurofilament subunits (NF-H and NF-M) and demonstrate their existence in the normal nervous system of adult rats. J Neurosci 7:3474-3488.
Li BS, Veeranna, Grant P, Pant HC (1999) Calcium influx and membrane depolarization induce phosphorylation of neurofilament (NF-M) KSP repeats in PC12 cells. Brain Res Mol Brain Res 70:84-91.

Lin LF, Doherty DH, Lile JD, Bektesh S, Collins F (1993) GDNF: a glial cell line-derived neurotrophic factor for midbrain dopaminergic neurons. Science 260:1130-1132.

Lubetzki C, Demerens C, Anglade P, Villarroya H, Frankfurter A, Lee VM, Zalc B (1993) Even in culture, oligodendrocytes myelinate solely axons. Proc Natl Acad Sci USA 90:6820-6824.

McCarthy KD, de Vellis J (1980) Preparation of separate astroglial and oligodendroglial cell cultures from rat cerebral tissue. J Cell Biol 85:890-902.

Meyer-Franke A, Kaplan MR, Pfrieger FW, Barres BA (1995) Characterization of signaling interactions that promote the survival and growth of developing retinal ganglion cell in culture. Neuron 15:805-819.

Nicole O, Ali C, Docagne F, Plawinski L, MacKenzie ET, Vivien D, Buisson A (2001) Neuroprotection mediated by glial cell line-derived neurotrophic factor: involvement of a reduction of NMDA-induced calcium influx by the mitogen-activated protein kinase pathway. J Neurosci 21:3024-3033.

Ohgoh M, Kimura M, Ogura H, Katayama K, Nishizawa Y (1998) Apoptotic death of cultured cerebral cortical neurons induced by withdrawal of astroglial trophic support. Exp Neurol 149:51-63.

Pang L, Sawada T, Decker SJ, Saltiel AR (1995) Inhibition of MAP kinase kinase blocks the differentiation of PC-12 cells induced by nerve growth factor. J Biol Chem 9:13585-13588.

Raff MC, Barres BA, Burne JF, Coles HS, Ishizaki Y, Jacobson MD (1994) Programmed cell death and the control of cell survival. Philos Trans R Soc Lond B Biol Sci 345:265-268.

Raff MC, Whitmore AV, Finn JT (2002) Axonal self-destruction and neurodegeneration. Science 296:868-871.

Riethmacher D, Sonnenberg-Riethmacher E, Brinkmann V, Yamaai T, Lewin GR, Birchmeier C (1997) Severe neuropathies in mice with targeted mutations in the ErbB3 receptor. Nature 389:725-730.

Saarma M, Sariola H (1999) Other neurotrophic factors: glial cell linederived neurotrophic factor (GDNF). Microsc Res Tech 45:292-302.

Sanchez I, Hassinger L, Paskevich PA, Shine HD, Nixon RA (1996) Oligodendroglia regulate the regional expansion of axon calibre and local accumulation of neurofilaments during development independently of myelin formation. J Neurosci 16:5095-5105.

Sanchez I, Hassinger L, Sihag RK, Cleveland DW, Mohan P, Nixon RA (2000) Local control of neurofilament accumulation during radial growth of myelinating axons in vivo: selective role of site-specific phosphorylation. J Cell Biol 151:1013-1024.

Snider WD (1994) Functions of the neurotrophins during nervous system development: what the knockouts are teaching us. Cell 77:627-638.

Strelau J, Unsicker K (1999) GDNF family members and their receptors: expression and functions in two oligodendroglial cell lines representing distinct stages of oligodendroglial development. Glia 26:291-301.

Trapp BD, Peterson J, Ransohoff RM, Rudick R, Mork S, Bo L (1998) Axonal transection in the lesions of multiple sclerosis. N Engl J Med 338:278-285.

Vaillant AR, Mazzoni I, Tudan C, Boudreau M, Kaplan DR, Miller FD (1999) Depolarization and neurotrophins converge on the phosphatidylinositol 3-kinase-Akt pathway to synergistically regulate neuronal survival. J Cell Biol 146:955-966.

Veeranna, Amin ND, Ahn NG, Jaffe H, Winters CA, Grant P, Pant HC (1998) Mitogen-associated protein kinases (Erk1,2) phosphorylate LysSer-Pro (KSP) repeats in neurofilament proteins NF-H and NF-M. J Neurosci 18:4008-4021.

Wilkins A, Chandran S, Compston A (2001) A role for oligodendrocytederived IGF-1 in trophic support of cortical neurons. Glia 36:48-57. 